\title{
The Use of Patient Reported Outcome Measures and Experiences in Patients With Anaemia Receiving Intravenous Ferric Carboxymaltose: A Cohort Study
}

Kerstin Wyssusek ( $\sim$ kwyssusek@gmail.com )

Royal Brisbane and Women's Hospital, Brisbane, Australia 2. The University of Queensland, Faculty of Medicine https://orcid.org/0000-0003-1709-4958

Cienwen Town

Royal Brisbane and Women's Hospital

Rose Norton

UQ Faculty of Medicine: The University of Queensland Faculty of Medicine

Annie Der Avedissian

UQ Faculty of Medicine: The University of Queensland Faculty of Medicine

\section{Research}

Keywords: Patient Reported Outcomes (PROs), Instruments used to evaluate the patient's own health, quality of life, functional status associated with the received health care or treatment, Health-Related Quality of Life Measures, Patient Reported Outcome Measures (PROMs), Patient Reported Experience Measures (PREMs)

Posted Date: September 15th, 2020

DOl: https://doi.org/10.21203/rs.3.rs-75415/v1

License: (c) (1) This work is licensed under a Creative Commons Attribution 4.0 International License. Read Full License 


\section{Abstract}

\section{Background:}

Fatigue due to iron deficiency anaemia (IDA) has not been regularly evaluated in clinical studies. The aim of this study is to assess patient reported outcomes amongst individuals diagnosed with IDA before and after receiving intravenous ferric carboxymaltose. Fatigue and satisfaction will be measured using the validated Functional Assessment of Chronic Illness Therapy - Fatigue (FACIT-fatigue) scale and the validated Nordic Patient Experiences Questionnaire (NORPEQ), respectively.

\section{Methods:}

Thirty eligible patients receiving IV ferric carboxymaltose at the Royal Brisbane and Women's Hospital were enrolled to participate in interviews; two patients were lost to follow up. Mean change in FACITfatigue score from the baseline day of IV ferric carboxymaltose treatment to week 4 post treatment was measured. Patient satisfaction with the overall process of treatment was measured on the day of treatments. Approval for Ethics exemption was obtained by the RBWH ethics committee.

\section{Results:}

A one-way ANOVA was conducted to compare the patient questionnaire results at baseline on the day of receiving the IV ferric carboxymaltose and after a four week follow up via telephone interview. There was a significant effect of improvement of FACIT-fatigue scores on follow up at the $p<.05$ level $[F(1,54),=7.60$, $p=.008]$. Patient satisfaction was assessed by analysing NORPEQ, an 8-item questionnaire. Patients had reported overall higher satisfaction scores at baseline and follow-up, averaging $91 \%$ and $94 \%$, respectively and were not significant in treatment at the $p<.05$ level $[F(1,52),=1.37, p=.246]$.

\section{Conclusions:}

The use of validated patient reported outcome measures and experiences provides valuable insights into the patients' perspective. Physiological and biochemical data outcomes are often used as the goldstandard of medical research. However, patient experience and self-perceived physiological improvement are imperative when measuring the success of a medical intervention and the benefits it provides to patients and consumers. Further research is needed to combine the subjective patient experience data with objective physical, biochemical, or physiological data. Combined, they can provide a thorough and comprehensive assessment of an intervention. Furthermore, a comparison of outcomes administering IV iron or oral iron therapy would provide valuable information about efficacy.

\section{Trial registration:}

Not registered

\section{Background}


The World Health Organization (WHO) defines anemia according to hemoglobin thresholds of $<120 \mathrm{~g} / \mathrm{L}$ in non-pregnant women and $<130 \mathrm{~g} / \mathrm{L}$ in men (3). Anemia is a global issue with significant health, social, and economic consequences. According to the WHO, the global prevalence of this blood disorder is 1.62 billion people, or $24.8 \%$ of the population (3). It is associated with increased morbidity and mortality, and is the leading impairment for males, and females in years lived with disability (YLD) according to the 2017 Global Burden of Disease report (4). This includes adverse effects on mental and psychomotor development in infants, increased risk of preterm birth, low birth weight, and infant and maternal mortality $(5,6,7)$. Anemia is also associated with increased risk of infection, and heart failure (8). Although there are many types of anemia, IDA is the most common and has a significant global burden, despite being the most treatable of all anemias (9). Additionally, IDA is associated with decreased quality of life, productivity, and physical functioning (10). It is also an independent risk factor for increased length of hospital stay and poor clinical outcomes in patients undergoing surgery (11).

The symptoms commonly seen in IDA may be due to the anemia itself or due to a deficiency in iron. These symptoms include fatigue, poor concentration, lack of endurance, tachycardia, pica, restless leg syndrome and hair loss (12).

Oral iron is considered the suitable first line therapy for iron deficiency and iron deficiency anemia except for conditions such as gastric bypass, heavy uterine bleeding, and inflammatory bowel disease $(13,14)$. Oral iron is also not recommended in those requiring rapid repletion of iron, such as in patients who are less than 2 months away from a non-deferrable surgery, because IDA further complicates the morbidity and mortality seen in perioperative patients. Even so, the efficacy of oral iron replacement may be hindered by patient compliance owing to its long treatment course and common side effects. Treatment with oral iron may take up to 6 months to replete iron stores $(15,16)$. Additionally, a significant number of patients report gastrointestinal side effects, which has been shown to hinder adherence to treatment (17). Adherence rates to oral iron range from $40-60 \%$ in patients with IDA (18). Other side effects of oral iron include constipation, diarrhea, metallic taste, and thick, green and tenacious stool. Additionally, endoscopies in patients taking oral iron often commonly shows ulcerative erosions and gastritis (19).

Even with strict compliance, iron absorption is not necessarily assured and can be affected by other factors. Ideally, oral iron should be taken on an empty stomach at least 1 hour prior to a meal with a source of vitamin $\mathrm{C}$ to maximize absorption. Moreover, substances that inhibit iron absorption such as fiber, milk, tannins (in tea or coffee), and stomach-acid inhibiting medications should be avoided several hours prior to taking oral iron.

Therefore, intravenous ferric carboxymaltose (Ferinject ${ }^{\circledR}$ ) is an effective option to correct iron deficiency anemia in patients for whom oral iron preparations are ineffective or cannot be administered.

\section{Patient Reported Outcomes in Clinical Research}

Patient-Reported Outcomes (PROs) are tools used to assess health experiences including how patients feel or function in relation to their health, disease, or treatment (20). PROs offer valuable insights into the 
patients' perspective that can provide a thorough and comprehensive assessment of an intervention when combined with objective physical, biochemical, or physiological data. Therefore, PROs can be used as primary or secondary trial endpoints in clinical trials (21).

The use of PRO measures in clinical trials has been increasing, particularly in oncology research where PROs provide valuable information on quality of life and adverse effects of chemotherapy treatments (22, 23). This trend will likely continue to increase with initiatives such as the Patient-Focused Drug Development and Food and Drug Administration (FDA) PRO Guidance (24).

The use of PROs is growing within clinical practice and has been associated with enhanced physician satisfaction, improved physician-patient relationships due to shared decision making, and increased workflow efficiency (25).

\section{The Ideal PRO instruments}

The PRO tools that are used must have a high content validity, which is defined as the extent to which an instrument measures the important aspect of concepts that developers or users purport it to assess (20). The importance of a high content validity is stressed by the United States (US) Food and Drug Administration and the European Medicines Agency (20).

The PRO instrument should also be specific to the concept being measured, should have an optimum number of items, and should have proper evidence for the conceptual framework it strives to measure. The PRO instrument should have strong content, construct and criterion validity. It should also have strong test-retest reliability, internal consistency and inter-interviewer reliability.

\section{PROs Used in Patients with IDA}

Staibano et al. conducted a scoping review of patient reported outcome measures used in the treatment of anemia based on studies published from 1990 to 2017 (26). Of the 3,224 studies identified, 130 met their eligibility criteria. PROs were specified as primary outcome measures in $25.4 \%$ of studies and as secondary outcome measures in $56.9 \%$ of studies. In $21.5 \%$ of studies PROs were reported but not specified as primary or secondary endpoints. In contrast, $40 \%$ of studies reported only laboratory outcomes, such as hemoglobin levels or number of patients reaching hemoglobin target as the primary end point.

Based on a review by Staibano et all. the most commonly used PROM was the Functional Assessment of Cancer Therapy (FACT) and Functional Assessment of Chronic Illness Therapy-Fatigue (FACIT-Fatigue) (26). FACT/FACIT-fatigue was originally developed to measure cancer-associated fatigue but has since been shown to assess fatigue and related symptoms on daily activities in various chronic conditions. The content validity and psychometric validity of the FACIT-Fatigue scale in IDA patients has been proven (27). It has also been proven in hospitalized patients with IDA (28).

The second most commonly used PROM is the Linear analogue scale assessment (LASA). LASA was most commonly used to assess quality of life in cancer patients and was psychometrically sound to use 
as a short questionnaire in cancer patients (29). However, no further studies could be found to demonstrate the psychometric validity in peri-operative patients with IDA.

The third most commonly used PROM is the 36 item short form survey (SF-36). This PROM is a validated instrument in anemic patients with chronic kidney disease (30). In a study conducted by Sim et al., the SF36 PROM was used in conjunction with other validated measures to assess preoperative anemia, functional outcomes and quality of life following hip fracture surgery (31).

The Kidney disease quality of life instrument (KDQOL) measure is designed to assess quality of life in patients with kidney disease. This PROM has a proven reliability and construct validity for assessing health-related quality of life among dialysis patients (32). The European Platform of Cancer Research (EORTC) Quality of Life of Cancer Patients questionnaire (QLQ C30) is effective in the measurement of quality of life in cancer patients, and the Kansas City Cardiomyopathy Questionnaire (KCCQ) questionnaire is a commonly used instrument to measure quality of life and health status for patients with heart failure (33).

\section{Measuring Patient Experiences instead of Patient Satisfaction Using Validated Patient Reported Experience Measures}

PREMs are used to measure patient experiences in the inpatient setting. There are a number of PREMs that have been shown to be valid and reliable. Smith et al. (2015) conducted a scoping review on the Patient-Reported Experience Measures to rank PREM tools based on validity and reliability (34). The Nordic Patient Experiences Questionnaire (NORPEQ) was shown to have strong reliability and validity (35).

\section{Study Aim:}

The aim of our study was to evaluate patient reported outcomes amongst individuals diagnosed with IDA before and after receiving intravenous ferric carboxymaltose. The severity of fatigue and patient satisfaction with treatment were assessed using the validated Functional Assessment of Chronic IIIness Therapy - Fatigue (FACIT-fatigue) scale and the validated Nordic Patient Experiences Questionnaire (NORPEQ) $(35,36)$. Additionally, we measured overall patient satisfaction and experiences during the intravenous procedure.

\section{Methods}

Thirty eligible patients receiving IV ferric carboxymaltose at the Royal Brisbane and Women's Hospital were enrolled to participate in interviews between January 2019 and October 2019. Patient eligibility was determined based on the following criteria: comprehension of spoken English; the ability to provide informed verbal consent; nil previous or recent (within $<2$ month) administration of IV ferric carboxymaltose; no contraindications to the administration of IV ferric carboxymaltose. No restrictions were made on patient demographics including gender, age and medical history. Two patients were lost to follow-up and removed from the study results. 
Patient demographics were collected via electronic patient record systems such as the Viewer and Integrated Electronic Medical Record (ieMR). Pathology results were also collected from private pathology providers, Sullivan and Nicolaides and Queensland Medical Laboratory (QML) pathology.

Mean change in FACIT-fatigue score from the baseline day of IV ferric carboxymaltose treatment to week 4 post treatment was measured. Eligible patients were asked to complete the FACIT-Fatigue scale (version 4) survey during their procedure. The survey included 13 questions assessing fatigue across a range of activities during the last seven days (Table 1). Responses are measured on a scale of zero to four, with zero being "not at all" and four being "very much." A score of less than 30 indicates severe fatigue. The higher the score, the better the quality of life.

Table 1

FACIT fatigue scale

\section{FACIT Fatigue Scale (Version 4)}

Below is a list of statements meant to gauge how tired you feel. Please indicate your response as it applied to the past 7 days.

\begin{tabular}{lllll}
$\begin{array}{l}\text { Not at } \\
\text { all }\end{array}$ & $\begin{array}{l}\text { A little } \\
\text { bit }\end{array}$ & $\begin{array}{l}\text { Some- } \\
\text { what }\end{array}$ & $\begin{array}{l}\text { Quite } \\
\text { a bit }\end{array}$ & $\begin{array}{l}\text { Very } \\
\text { much }\end{array}$ \\
\hline 0 & 1 & 2 & 3 & 4
\end{tabular}

HI7 I feel fatigued

HI12 I feel weak all over

An1 I feel listless ("washed out")

An2 I feel tired

An3 I have trouble starting things because I am tired

An4 I have trouble finishing things because I am tired

An5 I have energy

An7 I am able to do my usual activities

An8 I need to sleep during the day

An12 I am too tired to eat

An14 I need help doing my usual activities

An15 I am frustrated by being too tired to do the things I want to do

An16 I have to limit my social activity because I

am tired 
Patient satisfaction with the overall process of treatment was also measured on the day of treatment using the NORPEQ Questionnaire. The NORPEQ questionnaire is an 8-item survey with a higher score indicating a higher level of patient satisfaction (Table 2). All follow up interviews were conducted via telephone. Prior to study commencement, approval for Ethics exemption was obtained by the RBWH ethics committee (LNR/2019/QRBW/50352).

Table 2

NORPEQ Questionnaire

\section{NORPEQ Questionnaire}

Below are questions regarding your experience in the hospital

To what extent...

$\begin{array}{lllll}\begin{array}{l}\text { Not } \\ \text { at } \\ \text { all }\end{array} & \begin{array}{l}\text { To a } \\ \text { small } \\ \text { extent }\end{array} & \begin{array}{l}\text { To } \\ \text { some } \\ \text { extent }\end{array} & \begin{array}{l}\text { To a } \\ \text { large } \\ \text { extent }\end{array} & \begin{array}{l}\text { To a very } \\ \text { large } \\ \text { extent }\end{array} \\ 1 & 2 & 3 & 4 & 5\end{array}$

Were the doctors easy to understand?

Did you have confidence in the doctors' professional knowledge?

Did you have confidence in the nurses' professional knowledge?

Did the nurses take care of you?

Did the doctors and nurses show interest in you when you were describing your situation?

Did you receive sufficient information prior to examination?

Was the overall care and treatment you received at the hospital satisfactory?

Do you think that you in any way received the wrong medical treatment?

\section{Results}

\section{Demographics:}

The median age of patients in this study was 55.5 years; the youngest patient in the study was 23 and the oldest 90 (Table 3 ). The median body mass index was 31.5 with a range of 28.8 to 36.3 , which is categorized as obese according to the WHO definition. The majority of study participants were Caucasian (88.8\%), with an even distribution of gender. 
Table 3

Demographic data in iron transfusion patients with anaemia. IBD, inflammatory bowel disease (crohn's and ulcerative colitis); RA, rheumatoid arthritis; CD, celiac disease; SLE, systemic lupus erythematosus; $\mathrm{MCV}$, mean cell volume; TIBC, total iron binding capacity.

\begin{tabular}{|c|c|c|}
\hline Variable $(n=28)$ & $\begin{array}{l}\text { Number of } \\
\text { Patients }\end{array}$ & $\begin{array}{l}\text { Mean (SD)/ Median (Range)/ } \\
\text { Percentage (\%) }\end{array}$ \\
\hline Gender (female) & 15 & $53.5 \%$ \\
\hline Body Weight (kg) & 25 & $92.6(27.7)$ \\
\hline Body Mass Index $\left(\mathrm{kg} / \mathrm{m}^{2}\right)$ & 24 & $31.5(28.8-36.3)$ \\
\hline Age (years) & 28 & $55.5(44.5-73.3)$ \\
\hline \multicolumn{3}{|l|}{ Age } \\
\hline $21-30$ & 4 & $14.3 \%$ \\
\hline $41-50$ & 7 & $25.0 \%$ \\
\hline $51-60$ & 4 & $14.3 \%$ \\
\hline $61-70$ & 3 & $10.7 \%$ \\
\hline $71-80$ & 6 & $21.4 \%$ \\
\hline $81-90$ & 4 & $14.3 \%$ \\
\hline \multicolumn{3}{|l|}{ Ethnicity } \\
\hline White/ Caucasian & 25 & $88.8 \%$ \\
\hline Asian & 1 & $3.7 \%$ \\
\hline Maori/ Polynesian & 1 & $3.7 \%$ \\
\hline Other (Iranian) & 1 & $3.7 \%$ \\
\hline \multicolumn{3}{|l|}{ Co-morbidities } \\
\hline Chronic kidney disease & 12 & $42.9 \%$ \\
\hline $\begin{array}{l}\text { Diabetes mellitus (type } 1 \text { and type } \\
\text { 2) }\end{array}$ & 9 & $35.7 \%$ \\
\hline Ischaemic heart disease & 6 & $25.0 \%$ \\
\hline $\begin{array}{l}\text { Inflammatory diseases (IBD, RA, CD, } \\
\text { SLE ) }\end{array}$ & 6 & $21.4 \%$ \\
\hline Congestive cardiac failure & 4 & $14.3 \%$ \\
\hline Small bowel angioectasia & 4 & $14.3 \%$ \\
\hline Metastatic cancer & 3 & $10.7 \%$ \\
\hline
\end{tabular}




\begin{tabular}{|cll|}
\hline Variable $(\mathbf{n}=\mathbf{2 8})$ & $\begin{array}{l}\text { Number of } \\
\text { Patients }\end{array}$ & $\begin{array}{l}\text { Mean (SD)/Median (Range)/ } \\
\text { Percentage (\%) }\end{array}$ \\
\hline Nephrotic syndrome & 3 & $10.7 \%$ \\
\hline $\begin{array}{l}\text { Chronic obstructive pulmonary } \\
\text { disease }\end{array}$ & 2 & $7.1 \%$ \\
\hline Abnormal uterine bleeding & 2 & $7.1 \%$ \\
\hline Liver cirrhosis & 1 & $3.6 \%$ \\
\hline Medications & 10 & \\
\hline Antiarrhythmics & 8 & $35.7 \%$ \\
\hline Antihypertensives & 5 & $28.6 \%$ \\
\hline Proton-pump inhibitors & 1 & $17.9 \%$ \\
\hline Non-steroidal anti-inflammatories & & $3.6 \%$ \\
\hline Type of Patient & 21 & $75 \%$ \\
\hline Medical & 7 & $25 \%$ \\
\hline Surgical & & \\
\hline
\end{tabular}

There was a large range of common co-morbidities found in these patients, the most prevalent being chronic kidney disease, which was present in $42.9 \%$ of participants (Fig. 1). The most prevalent medication group in study participants was anti-hypertensives (35.7\%), followed by anti-arrhythmics $(28.6 \%)$. The majority of patients included in this study were medical patients (75\%) as opposed to surgical patients $(25 \%)$.

\section{PROM/PREM results}

A one-way ANOVA between subjects was conducted to compare the patient questionnaire results at baseline on the day of receiving the IV ferric carboxymaltose and after a four week follow up via telephone interview (Table 4). Two patients were lost to follow-up and removed from analysis. 
Table 4

One-way ANOVA comparing FACIT scores pre iron infusion to 4 weeks post infusion

\begin{tabular}{|c|c|c|c|c|c|c|}
\hline & Facit-Pre & Facit-Post & Difference & Norpeq-pr & Norpeq-po & Difference \\
\hline Patient 1 & 39 & 44 & 5 & 35 & 39 & 4 \\
\hline Patient 2 & 22 & 35 & 13 & 39 & 39 & 0 \\
\hline Patient 3 & 40 & 44 & 4 & 32 & 39 & 7 \\
\hline Patient 4 & 44 & 44 & 0 & 39 & 39 & 0 \\
\hline Patient 5 & 13 & 44 & 31 & 39 & 39 & 0 \\
\hline Patient 6 & 28 & 22 & -6 & 38 & 39 & 1 \\
\hline Patient 7 & 17 & 30 & 13 & 39 & 39 & 0 \\
\hline Patient 8 & 22 & 39 & 17 & 39 & 39 & 0 \\
\hline Patient 9 & 30 & 37 & 7 & 39 & 39 & 0 \\
\hline Patient 10 & 17 & 34 & 17 & 39 & 38 & -1 \\
\hline Patient 11 & 39 & 44 & 5 & 34 & 38 & 4 \\
\hline Patient 12 & 14 & 23 & 9 & 37 & 39 & 2 \\
\hline Patient 13 & 41 & 40 & -1 & 29 & 28 & -1 \\
\hline Patient 14 & 10 & 31 & 21 & 38 & 39 & 1 \\
\hline Patient 15 & 19 & 44 & 25 & & & 0 \\
\hline Patient 16 & 36 & 38 & 2 & 39 & 39 & 0 \\
\hline Patient 17 & 43 & 44 & 1 & 39 & 39 & 0 \\
\hline Patient 18 & 38 & 38 & 0 & 39 & 39 & 0 \\
\hline Patient 19 & 49 & 51 & 2 & 27 & 31 & 4 \\
\hline Patient 20 & 47 & 47 & 0 & 39 & 39 & 0 \\
\hline Patient 21 & 34 & & -34 & 36 & & -36 \\
\hline Patient 22 & 18 & & -18 & 24 & & -24 \\
\hline Patient 23 & 39 & 42 & 3 & 39 & 39 & 0 \\
\hline Patient 24 & 44 & 40 & -4 & 39 & 39 & 0 \\
\hline Patient 25 & 26 & 25 & -1 & 39 & 39 & 0 \\
\hline Patient 26 & 52 & 52 & 0 & 35 & 36 & 1 \\
\hline
\end{tabular}




\begin{tabular}{|lllllll|}
\hline & Facit-Pre & Facit-Post & Difference & Norpeq-pr & Norpeq-po & Difference \\
\hline Patient 27 & 16 & 43 & 27 & 39 & 39 & 0 \\
\hline Patient 28 & 19 & 33 & 14 & 37 & 34 & -3 \\
\hline Patient 29 & 34 & 37 & 3 & 30 & 35 & 5 \\
\hline Patient 30 & 22 & 36 & 14 & 36 & 39 & 3 \\
\hline AVERAGE & 28.9 & 37.60714 & & 36.3 & 37.8 & \\
\hline StDev & 13.2 & 9.1 & & 4.1 & 2.8 & \\
\hline StError & 2.4 & 1.7 & & 0.8 & 0.5 & \\
\hline
\end{tabular}

There was a significant effect of improvement of FACIT-fatigue scores on follow up at the $p<.05$ level $[F(1,54),=7.60, p=.008]$. Patient satisfaction was assessed by analysing NORPEQ, an 8 item survey in which a higher score correlates with higher patient satisfaction. Patients had reported overall higher satisfaction scores at baseline and follow-up, averaging $91 \%$ and $94 \%$, respectively and were not significant in treatment at the $p<.05$ level $[F(1,52),=1.37, p=.246]$. All patients said they would go through the procedure of getting an iron infusion again. In response to a question about whether they noticed a difference physically after receiving the infusion, participants had many positive comments to say, such as "yes, I can now walk up the stairs without stopping" and "yes, I feel less dizzy".

\section{Discussion}

The goal of this study was to evaluate patient reported outcomes before and after receiving intravenous ferric carboxymaltose in individuals who had been diagnosed with iron deficiency anaemia. This study found that participants experienced a significant improvement in perceived functional capacity at 4 weeks post IV ferric carboxymaltose administration. This response correlates with the anticipated time for IV iron uptake and synthesis within the body (37). Other studies have found a similar improvement in patient reported fatigue after an intravenous iron infusion $(26,38,39,40,41)$. Darlington et al. (2008) found that exercise capacity and symptoms improved after an iron infusion in patients with congestive cardiac failure (CCF) and iron deficiency, and that this effect was more pronounced if the patient was also anaemic (37). Bolger et al. (2006) also reported that intravenous iron infusion improved exercise capacity and reduced symptoms in CCF patients (42). Holm et al. (2018) found a statistically significant reduction in fatigue after intravenous iron isomaltoside in women who have had a postpartum haemorrhage (38). Similarly, Seid (2014) found that iron infusions in women with abnormal uterine bleeding and IDA resulted in a significant improvement in self-reported quality of life (39).

None of the study participants experienced any adverse side effects relating to the treatment, and all participants conveyed confidence in their understanding of why they were receiving the treatment and its intended purpose. All participants felt they were receiving the appropriate treatment for their underlying conditions, leading researchers to believe that participants had been well educated prior to their treatment 
and that they had an appropriate level of trust in their treating physicians and nursing staff. Interestingly, a randomized trial of intravenous and oral iron therapy in chronic kidney disease patients found that IV iron therapy was associated with an increased risk of serious adverse events (43). However, this result was not replicated in other literature $(26,38,39,40,42,44)$.

Most studies on the benefits of iron infusions are targeted at a specific patient sub-group, the age, gender and ethnicity profile of patients in this study are comparable to similar research $(2,11,26,27,46)$. Other studies into iron deficiency anaemia identified a similar list of patient co-morbidities as those recorded by this study. Carson et al. (2016) found that only $11.3 \%$ of patients had a history of cardiovascular disease; $9.8 \%$ of patients had diabetes mellitus; and they did not record whether patients had CKD (47). In contrast, this study found $25 \%$ of patients to have ischaemic heart disease (IHD) and $35.7 \%$ had diabetes mellitus (DM). Carson et al. (2016) exclusively evaluated surgical anaemic patients, whereas $75 \%$ of the patient cohort in this study were medical patients who often have more comorbidities, this could account for the higher rates of IHD and DM (46). Acaster et al. (2015) conducted research to validate the FACITfatigue scale as a measurement of fatigue in iron deficiency anaemia patients (27). They found that $42.8 \%$ of their patient population had the co-morbidity of abnormal uterine bleeding (AUB), in comparison to this study which found only $7.1 \%$ of patients had this condition (27). Acaster et al. (2015) also found $4.8 \%$ of their patients to have cancer and $28.5 \%$ of their patients to have GI disorders (27). This study only recorded metastatic cancer patients (10.7\%) and GI disorders have been included in inflammatory conditions, which includes rheumatoid arthritis and systemic lupus erythematosus as well as inflammatory bowel disease and celiac disease (21.4\%).

Chronic kidney disease (CKD) was found to be the most prevalent co-morbidity among the patient cohort (42.9\%). Iron deficiency anaemia is a common complication of chronic kidney disease $(47,48,49)$. There are many factors which contribute to iron deficiency in CKD patients, these include poor oral intake of iron rich foods, increased iron utilization, poor iron absorption from the gastrointestinal tract, and proteinuria resulting in the loss of iron-containing proteins (48). Effectively managing anaemia in CKD patients has been demonstrated to improve quality of life, improve mortality rates, reduce rates of hospitalization and increase physical performance and exercise capacity $(49,50)$. Well known symptoms of anaemia, such as shortness of breath, dizziness and fatigue are associated with more severe adverse outcomes in CKD patients, such as cardiovascular complications including congestive heart failure (CCF) (49). This study found $14.3 \%$ of patients had congestive cardiac failure as a comorbidity. Interestingly, Ebner et al (2016) reported that congestive cardiac failure patients with anaemia most often present with CKD and hypertension (51). Iron deficiency has been shown to be an independent predictor of poor quality of life and mortality in CCF patients $(51,52)$. There are several factors that contribute to CCF patients developing iron deficiency anaemia, these include poor nutrition, malabsorption and gastrointestinal bleeding due to the use of oral anticoagulants and aspirin (53). Even in the absence of anaemia, many chronic diseases have been shown to worsen in the context of iron deficiency (53).

Patient reported outcome measures have the potential to provide valuable data pertaining to clinical practice and potential areas for improvement. Multiple review papers have assessed the use of patient 
reported outcomes in patients receiving iron infusions for IDA and have concluded that PROMs have only been sporadically assessed $(26,45)$. Staibano et al $(2018)$ found that only a minority of studies have patient reported outcomes as a primary objective, and that most studies have only mentioned patient reported outcomes in passing in the discussion (26). In this study, the FACIT-fatigue and NORPEQ tools provided a better understanding of the perceived benefits patients experience post IV ferric carboxymaltose treatment, and the high satisfaction response suggests that the existing treatment pathways in the hospital are consumer focused and inclusive.

Limitations of this study include the lack of comparable physiological or biological data to accompany the improved FACIT- fatigue scores, and the small sample size. Further research could collect blood samples before and after iron infusion and compare the pathology results to patient reported outcomes. Another limitation of this study is that there was no control group, previous literature into patient reported outcomes after iron infusions show there is a significant placebo effect (45). This study did not analyze whether fatigue scores improved more in co-morbid patients compared to patients without other significant medical history, future studies could assess whether iron infusions are more beneficial in patients with significant co-morbidities. This study used the NORPEQ score to measure patient satisfaction. The NORPEQ score was chosen because it was shown to have strong validity and reliability, however it was only previously been used in Nordic countries (35). Since this study selected the NORPEQ score, a new emerging PREM, called the Picker Patient Experience (PPE-15) questionnaire, was used to measure patient experience in Australia (58). The PPE-15 has also been shown to have a high degree of internal reliability and consistency in a study of acute care hospitals in 5 countries (UK, US, Germany, Sweden and Switzerland (58). Future Australian studies looking into patient satisfaction could use different PREM tools, such as the PPE-15.

\section{Conclusion}

The use of validated patient reported outcome measures and experiences provides valuable insights into the patients' perspective. Physiological and biochemical data outcomes are often used as the goldstandard of medical research. However, patient experience and self-perceived physiological improvement are imperative when measuring the success of a medical intervention and the benefits it provides to patients and consumers. In this study, patient reported outcomes were used to assess fatigue and patient satisfaction before and four weeks after receiving IV ferric carboxymaltose. Patients showed significant improvement in fatigue measurements following IV therapy and were overall satisfied with the procedure. Further research is needed to combine the subjective patient experience data with objective physical, biochemical, or physiological data. Combined, they can provide a thorough and comprehensive assessment of an intervention. Furthermore, a comparison of outcomes administering various formulas of IV iron or oral iron therapy would provide valuable information about efficacy.

\section{List Of Abbreviations}

Abnormal uterine bleeding AUB 
Analysis of variance ANOVA

Chronic kidney disease CKD

Congestive cardiac failure CCF

Diabetes mellitus DM

European Platform of Cancer Research EORTC

Food and drug administration FDA

Gastrointestinal GI

Hemoglobin $\mathrm{Hb}$

Functional Assessment of Cancer Therapy FACT

Functional assessment of chronic illness therapy -fatigue scale FACIT-fatigue scale

IDA Iron deficiency anaemia

Integrated Electronic Medical Record ieMR

Intravenous IV

Intravenous ferric carboxymaltose Ferinject ${ }^{\circledR}$

Ischaemic heart disease IHD

Kansas City Cardiomyopathy Questionnaire KCCQ

Kidney disease quality of life instrument KDQOL

Linear analogue scale assessment LASA

Nordic Patient Experiences Questionnaire NORPEQ

Patient Reported Outcomes PROs

Patient Reported Outcome Measures PROMs

Quality of Life of Cancer Patients QLQ C30

Queensland Medical Laboratory QML pathology

Royal Brisbane and Women's Hospital RBWH 
United Kingdom UK

United States US

World Health Organisation WHO

Years lived with disability YLD

36 item short form survey SF-36

\section{Declarations}

\section{Ethics approval and consent to participate}

The RBWH Human Research Ethics Committee (HREC) deemed that this study did not need HREC review as it met the categories of activates that are considered to be quality assurance or quality improvement activities and was consistent with the guidance provided in the in the document: 'Ethical Considerations in Quality Assurance and Evaluation Activites' 2014 published by the National Health and Medical Research Council. Therefore, it was exempt from HREC review and registered under:

LNR/2020/QRBW/64173.

\section{Consent for publication}

Consent was obtained from all participants in this research project.

\section{Availability of data and materials}

The datasets used and/or analyzed during the current study are available from the corresponding author on reasonable request.

\section{Competing interests}

The authors declare that they have no competing interests.

\section{Funding}

No funding was for this study.

\section{Authors' contributions}


KW Initial concept, protocol design, ethics application, interpretation of statistical analysis and writing the manuscript, CT protocol design, ethics application, writing the manuscript, AA literature review, data collection, interpretation of statistical analysis, writing the manuscript, RN data collection, writing the manuscript. All authors read and approved the final manuscript.

\section{Acknowledgements}

The authors would like to acknowledge staff at the Internal Medicine Day Therapy Unit at RBWH who administered the IV iron infusions.

\section{Authors information}

$\mathrm{KW}$ is the Director of the Department of Anaesthesia and Perioperative Medicine, Royal Brisbane and Women's Hospital and an Associate Professor of The University of Queensland. CT is a clinical nurse consultant and involved in perioperative anaemia management at RBWH. RN and AA are medical students at the University of Queensland.

\section{References}

1. Weldring T, Smith SMS. Patient-Reported Outcomes (PROs) and Patient-Reported Outcome Measures (PROMs). Health services insights. 2013;6:61-8.

2. Kingsley C, Patel S. Patient-reported outcome measures and patient-reported experience measures. BJA Education. 2017;17(4):137-44.

3. WHO. Worldwide prevalence on anaemia 1993-2005 [Internet]. [Available from: http://www.who.int/vmnis/database/anaemia/anaemia_status_summary/en/.

4. James SL, Abate D, Abate KH, Abay SM, Abbafati C, Abbasi N, et al. Global, regional, and national incidence, prevalence, and years lived with disability for 354 diseases and injuries for 195 countries and territories, 1990-2017: a systematic analysis for the Global Burden of Disease Study 2017. The Lancet. 2018;392(10159):1789-858.

5. Walter T, De Andraca I, Chadud P, Perales CG. Iron deficiency anemia: adverse effects on infant psychomotor development. Pediatrics. 1989;84(1):7-17.

6. Sukrat B, Wilasrusmee C, Siribumrungwong B, McEvoy M, Okascharoen C, Attia J, et al. Hemoglobin concentration and pregnancy outcomes: a systematic review and meta-analysis. BioMed research international. 2013;2013:769057.

7. Camaschella C. Iron deficiency. Blood. 2018.

8. Anand IS, Gupta P. Anemia and Iron Deficiency in Heart Failure: Current Concepts and Emerging Therapies. Circulation. 2018;138(1):80-98.

9. Bruno de Benoist EM, Egli I, Mary Cogswell Worldwide prevalence of anaemia 1993-2005 [Internet]. 2008 [Available from: 
http://apps.who.int/iris/bitstream/handle/10665/43894/9789241596657_eng.pdf?sequence=1.

10. Kassebaum NJMD. The Global Burden of Anemia. Hematology/Oncology Clinics of North America. 2016;30(2):247-308.

11. Baron DM, Hochrieser H, Posch M, Metnitz B, Rhodes A, Moreno RP, et al. Preoperative anaemia is associated with poor clinical outcome in non-cardiac surgery patients. $\mathrm{Br} \mathrm{J}$ Anaesth. 2014;113(3):416-23.

12. DeLoughery TGMDMF. Iron Deficiency Anemia. Med Clin North Am. 2017;101(2):319-32.

13. Auerbach M, Adamson JW. How we diagnose and treat iron deficiency anemia. Am J Hematol. 2015;91(1):31-8.

14. Prepoerative Anaemia Identification, Assessment and Management. Case Study: National Blood Authority Australia June 2014 [updated June 2014. Available from:

https://www.blood.gov.au/system/files/documents/preoperative-anaemia-identification-assessmentand-management-case-study\%20grey\%20cover\%20v4.pdf.

15. Bhandari S, Pereira ID, Chappell FH, Drakesmith H. Intravenous Irons: From Basic Science to Clinical Practice. Pharmaceuticals. 2018;11(3).

16. Baird-Gunning J, Bromley J. Correcting iron deficiency. Australian prescriber. 2016;39(6):193-9.

17. Tolkien Z, Stecher L, Mander AP, Pereira DIA, Powell JJ. Ferrous sulfate supplementation causes significant gastrointestinal side-effects in adults: a systematic review and meta-analysis. PloS one. 2015;10(2):e0117383-e.

18. Cancelo-Hidalgo MJ, Castelo-Branco C, Palacios S, Haya-Palazuelos J, Ciria-Recasens M, Manasanch J, et al. Tolerability of different oral iron supplements: a systematic review. Curr Med Res Opin. 2013;29(4):291-303.

19. Kaye P, Abdulla K, Wood J, James P, Foley S, Ragunath K, et al. Iron-induced mucosal pathology of the upper gastrointestinal tract: a common finding in patients on oral iron therapy. Histopathology. 2008;53(3):311-7.

20. Patrick DL, Burke LB, Gwaltney CJ, Leidy NK, Martin ML, Molsen E, et al. Content ValidityEstablishing and Reporting the Evidence in Newly Developed Patient-Reported Outcomes (PRO) Instruments for Medical Product Evaluation: ISPOR PRO Good Research Practices Task Force Report: Part 1-Eliciting Concepts for a New PRO Instrument. Value in Health. 2011;14(8):967-77.

21. Mercieca-Bebber R, King MT, Calvert MJ, Stockler MR, Friedlander M. The importance of patientreported outcomes in clinical trials and strategies for future optimization. Patient related outcome measures. 2018;9:353-67.

22. Fromme EK, Eilers KM, Mori M, Hsieh Y-C, Beer TM. How Accurate Is Clinician Reporting of Chemotherapy Adverse Effects? A Comparison With Patient-Reported Symptoms From the Quality-ofLife Questionnaire C30. J Clin Oncol. 2004;22(17):3485-90.

23. Quinten C, Maringwa J, Gotay CC, Martinelli F, Coens C, Reeve BB, et al. Patient self-reports of symptoms and clinician ratings as predictors of overall cancer survival. J Natl Cancer Inst. 2011;103(24):1851-8. 
24. Vodicka E, Kim K, Devine EB, Gnanasakthy A, Scoggins JF, Patrick DL. Inclusion of patient-reported outcome measures in registered clinical trials: Evidence from ClinicalTrials.gov (2007-2013). Contemporary Clinical Trials. 2015;43:1-9.

25. Rotenstein LS, Huckman RS, Wagle NW. Making Patients and Doctors Happier - The Potential of Patient-Reported Outcomes. N Engl J Med. 2017;377(14):1309-12.

26. Staibano P, Perelman I, Lombardi J, Davis A, Tinmouth A, Carrier M, et al. Patient-Centered Outcomes in the Management of Anemia: A Scoping Review. Transfusion Medicine Reviews. 2018.

27. Acaster S, Dickerhoof R, DeBusk K, Bernard K, Strauss W, Allen LF. Qualitative and quantitative validation of the FACIT-fatigue scale in iron deficiency anemia. Health Qual Life Outcomes. 2015;13:60-.

28. Prochaska MT, Newcomb R, Block G, Park B, Meltzer DO. Association Between Anemia and Fatigue in Hospitalized Patients: Does the Measure of Anemia Matter? J Hosp Med. 2017;12(11):898-904.

29. Locke DEC, Decker PA, Sloan JA, Brown PD, Malec JF, Clark MM, et al. Validation of single-item linear analog scale assessment of quality of life in neuro-oncology patients. J Pain Symptom Manag. 2007;34(6):628-38.

30. Finkelstein FO, van Nooten F, Wiklund I, Trundell D, Cella D. Measurement properties of the Short Form-36 (SF-36) and the Functional Assessment of Cancer Therapy - Anemia (FACT-An) in patients with anemia associated with chronic kidney disease. Health Qual Life Outcomes. 2018;16(1):111-.

31. Sim YE, Sim S-eD, Seng C, Howe TS, Koh SB, Abdullah HR. Preoperative Anemia, Functional Outcomes, and Quality of Life After Hip Fracture Surgery. J Am Geriatr Soc. 2018;66(8):1524-31.

32. Peipert JD, Bentler PM, Klicko K, Hays RD. Psychometric Properties of the Kidney Disease Quality of Life 36-Item Short-Form Survey (KDQOL-36) in the United States. Am J Kidney Dis. 2018;71(4):4618.

33. 33. Masterson Creber R, Polomano R, Farrar J, Riegel B. Psychometric properties of the Kansas City Cardiomyopathy Questionnaire (KCCQ). Eur J Cardiovasc Nurs. 2012;11(2):197-206.

34. Smith A, Hex N, Taylor MT. Patient-Reported Experience Measures (PREMS) A Scoping Document to Inform the Evaluation of the NHS Vanguard Sites. 24. Wakefield: York Health Economic Consortium; 2015 Sep. 15p.

35. Skudal KE, Garratt AM, Eriksson B, Leinonen T, Simonsen J, Bjertnaes OA. The Nordic Patient Experiences Questionnaire (NORPEQ): cross-national comparison of data quality, internal consistency and validity in four Nordic countries. BMJ Open. 2012;2(3).

36. Yellen SB, Cella DF, Webster K, Blendowski C, Kaplan E. Measuring fatigue and other anemia-related symptoms with the Functional Assessment of Cancer Therapy (FACT) measurement system. J Pain Symptom Manage. 1997;13(2):63-74.

37. Okonko DO, Grzeslo A, Witkowski T, Mandal AKJ, Slater RM, Roughton M, Foldes Gabor, Thum T, Majda J, Banasiak W, Missouris CG, Poole-Wilson PA, Anker SD, Ponikowski P. Effect of intravenous iron sucrose on exercise tolerance in anemic and nonanemic patients with symptomatic chronic 
heart failure and iron deficiency: FERRIC-HF: A randomised, controlled, observer-blinded trial. J Am Coll Cardiol. 2008;51(2):103-12.

38. Holm C, Thomsen LL, Langhoff-Roos J. Intravenous iron isomaltoside treatment of women suffering from severe fatigue after postpartum haemorrhage. The journal of maternal-fetal and neonatal medicine. 2019; 32(17).

39. Seid MV, Dahl NV, Pharm D, Lau G, Bernard K, Strauss W. Effect of ferumoxytol on quality of life in iron deficiency anemia from abnormal uterine bleeding. Obstet Gynecol. 2014;1223(1):181-2.

40. Strauss W, Auerbach M. Health-related quality of life in patients with iron deficiency anemia: impact of treatment with intravenous iron. Patient related outcome measures. 2018;9:285-98.

41. Brookes M, Keeler B, Ng O, Padmanabhan H, Simpson A, Acheson A. PTU-110 The use of preoperative intravenous iron improves post-operative patient reported quality of life in anaemic colorectal cancer patients: results from a multicentre randomised control trial. BMJ Journals Online. 2017;66:A105.

42. Bolger AP, Bartlett FR, Penston HS, O'leary J, Pollock N, Kaprielian R, Chapman CM. Intravenous iron alone for the treatment of anemia in patients with chronic heart failure. J Am Coll Cardiol. 2006;48:1225-7.

43. Agarwal R, Kusek JW, Pappas MK. A randomised trial of intravenous and oral iron in chronic kidney disease. Kidney Int. 2015;88(4):905-14.

44. Madhu SV, Raj A, Gupta S, Giri S, Rusia U. Effect of iron deficiency anemia and iron supplementation on $\mathrm{HbA1c}$ levels - Implications for diagnosis of prediabetes and diabetes mellitus in Asian Indians. Clin Chim Acta. 2017;468:225-9.

45. Malinowski AK, D'Souza R, Khan KS, Shehata N, Malinowski M, Daru J. Reported outcomes in perinatal iron deficiency anemia trials: a systematic review. Gynecol Obstet Invest. 2019;84(5):41734.

46. Carson JL, Duff A, Poses RM, Berlin JA, Spence RK, Trout R, Noveck H. Effect of anaemia and cardiovascular disease on surgical mortality and morbidity. The Lancet. 1996;348:1055-60.

47. Farooque MU, Bhushan B. Anaemia and chronic kidney disease. Journal of Evidenced Based Medicine heathcare. 2017;4(39):2352-4.

48. Bennet L. Patient Independence in Chronic Kidney Disease and Anaemia: Implications of the 2012 KDIGO Guidelines. Journal of Renal Care. 2013;39(2):108-17.

49. O'Mara NB. Anemia in Patients with Chronic Kidney Disease. Diabetes Spectrum. 2008;21(1):12-9.

50. Murphy F, Bennett L, Jenkins K. Managing anaemia of chronic kidney disease. British Journal of Nursing. 2010;19(20):1281-6.

51. Ebner N, Jankowski P, Lainscak M, Elsner S, Sliziuk V, Steinbeck L, Kube J, Bekfani T, Scherbakov N, Valentova M, Sandek A, Doehner W, Springer J, Anker S, von Haekling S. The impact of iron deficiency and anaemia on exercise capacity and outcomes in patients with chronic heart failure. Results from the stdies investigating co-morbidities aggravating heart failure. Int $\mathrm{J}$ Cardiol. 2016;205:6-12. 
52. Palleschi L, Nunziata E. Iron deficiency and iron deficiency anaemia in elderly patients with chronic heart failure. Geriatric Care. 2017; 3(4).

53. Cohen-Solal A, Leclercq C, Deray G, Lasocki S, Zambrowski J, Mebazaa A, de Groote P, Damy T, Galinier M. Iron deficiency: an emerging therapeutic target in heart failure. BMJ Journals Heart. 2014; 100(18).

54. Firkin F, Rush B. Interpretation of biochemical tests for iron deficiency: diagnostic difficulties related to limitations of individual tests [Internet]. Strawberry Hills, NSW: Australian Prescriber, and independent review; 1997 [cited 13/07/2020]. Available from: https://www.nps.org.au/australianprescriber/articles/interpretation-of-biochemical-tests-for-iron-deficiency-diagnostic-difficultiesrelated-to-limitations-of-individual-tests.

55. Verdon F, Burnand B, Stubi CL, Bonard C, Graff M, Michaud A, Bischoff T, de Vevey M, Studer J, Herzig L, Chapuis C, Tissot J, Pecoud A, Favrat B. Iron supplementation for unexplained fatigue in nonanaemic women: double blind randomised placebo controlled trial. BMJ. 2003;326(7399):1124.

56. Vaucher P, Druais PL, Waldvogel S, Favrat B. Effect of iron supplementation on fatigue in nonanemic menstruating women with low ferritin: a randomized controlled trial. CMAJ. 2012;184(11):1247-54.

57. Macdougall IC. Iron supplementation in the non-dialysis chronic kidney disease (ND-CKD) patient: oral or intravenous. Curr Med Res Opin. 2010;26(2):473-82.

58. Harrison R, Walton M, Kelly P, Manias E, Jorm C, Smith-Merry J, et al. Hospitalization from the patient perspective: a data linkage study of adults in Australia. Int J Qual Health Care. 2018;30(5):358-65.

\section{Figures}

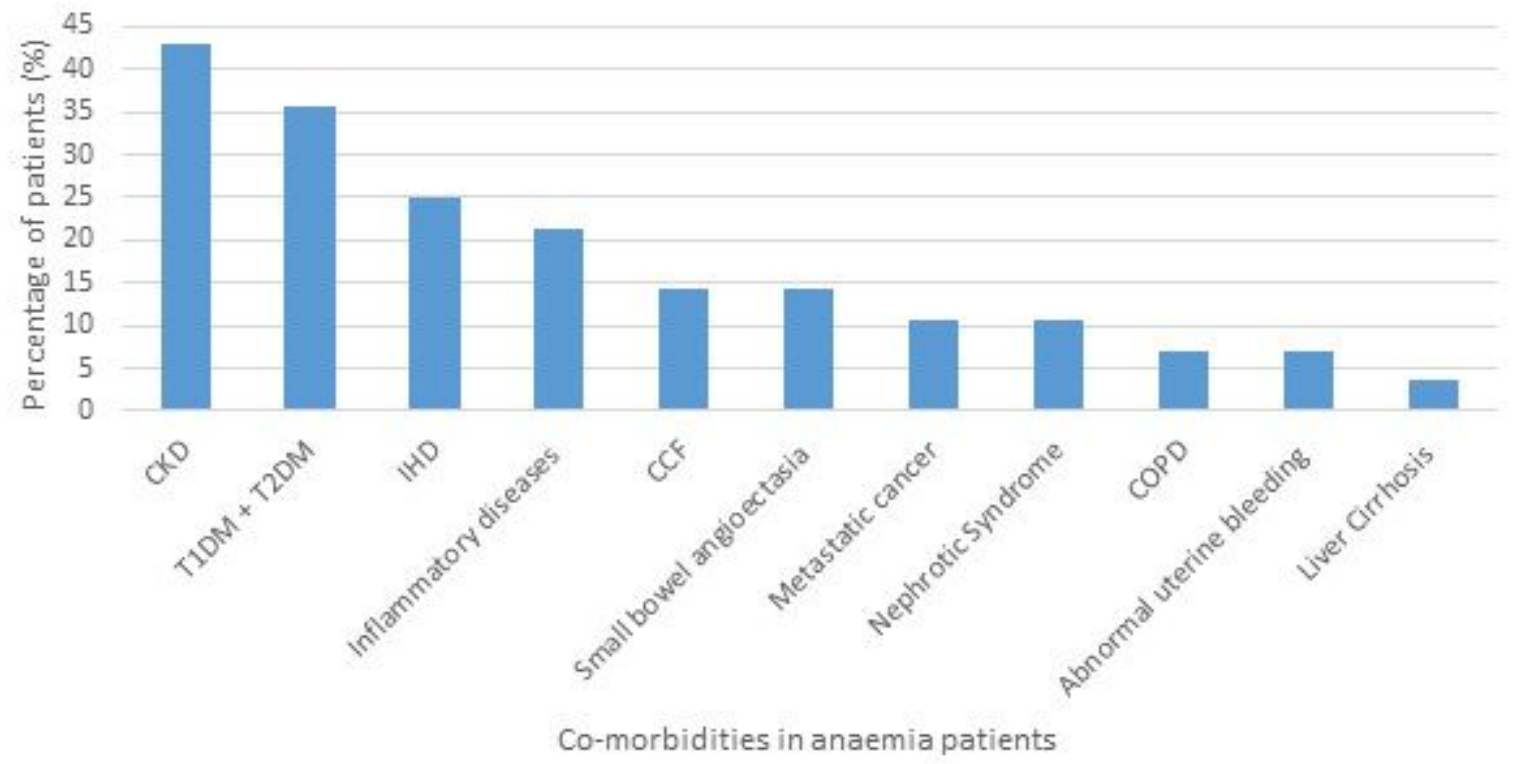

Figure 1 
Co-morbidities in iron transfusion patients with anaemia. CKD, Chronic kidney disease; T1DM + T2DM, type 1 diabetes mellitus and type 2 diabetes mellitus; IHD, ischaemic heart disease; inflammatory diseases in this study were inflammatory bowel diseases (crohn's disease and ulcerative colitis), rheumatoid arthritis, celiac disease and systemic lupus erythematosus; CCF, congestive cardiac failure; metastatic cancers in this study were metastatic rectal adenocarcinoma, metastatic melanoma, metastatic prostate cancer; COPD, chronic obstructive pulmonary disease. 